\title{
Randomized controlled trial to evaluate the utility of suction and inner-stylet of EBUS- TBNA for mediastinal and hilar lymphadenopathy
}

Xiaoxiao Lin ${ }^{1}$, Min Ye ${ }^{1}$, Yuping Li ${ }^{1}$, Jing Ren ${ }^{1}$, Qiyan Lou', Yangyang Li ${ }^{2}$, Xiaohui $\mathrm{Jin}^{3}$, Ko-Pen Wang ${ }^{4}$ and Chengshui Chen ${ }^{1 *}$ (D)

\begin{abstract}
Background: The optimal procedure for maximizing the diagnostic yield and minimizing the procedural complexity of endobronchial ultrasound-guided transbronchial needle aspiration (EBUS-TBNA) is controversial. We conducted a prospective randomized controlled trial to determine the optimal procedure of EBUS-TBNA for mediastinal and hilar lymphadenopathy, with a particular focus on the roles of the inner-stylet and suction.
\end{abstract}

Methods: Consecutive patients with enlarged mediastinal and hilar lymph nodes (LNs), detected by computed tomography $(C T)$ or positron emission tomography-CT (PET-CT), who underwent EBUS-TBNA were included. Each LN was sampled with three needle passes using suction-stylet, suction-no stylet, and stylet-no suction procedures. The samples were smeared onto glass slides for cytological evaluation. A single, blinded cytopathologist evaluated each set of slides. The primary outcomes were cytological specimen adequacy rate and diagnostic yield of malignant LNs. The secondary outcomes were tissue-core acquisition rate, procedural time, and the amount of bleeding.

Results: This study evaluated 97 patients with a total of 255 LNs. The final LN diagnosis was benign in 144, malignant in 104, and inadequate in 7 cases. There were no significant differences among the suction-stylet, suction-no stylet, and stylet-no suction groups in specimen adequacy rate $(87.1,88.2,85.9 \%$, respectively) or diagnostic yield of malignancy $(32.2,31.8,31.0 \%$, respectively). However, the use of suction was associated with an increase in tissue-core acquisition rate $(P<0.001)$. The no-stylet procedure decreased the average procedural time by $14 \mathrm{~s}(P<0.001)$. There was no significant difference in the amount of bleeding among the procedures.

Conclusions: The use of suction or non-use of an inner-stylet does not make a significant difference in cytological specimen adequacy or diagnostic yield when performing EBUS-TBNA. While omitting the stylet can simplify the procedure, applying suction can increase the tissue-core acquisition rate. These findings may assist endoscopic physicians in determining the optimal EBUS-TBNA procedure and warrant clinical verification in a future multicentre study.

Trial registration: Trial registration: (ChiCTR-IOR-17010616). Retrospective registered date: 12th February, 2017.

Keywords: Endobronchial ultrasound-guided transbronchial needle aspiration, Clinical trial, Lymphadenopathy, Malignant

\footnotetext{
* Correspondence: wzchencs@163.com

${ }^{1}$ Department of Pulmonary and Critical Care Medicine, The First Affiliated

Hospital of Wenzhou Medical University, Ouhai District, Wenzhou, China

Full list of author information is available at the end of the article
}

(c) The Author(s). 2018 Open Access This article is distributed under the terms of the Creative Commons Attribution 4.0 International License (http://creativecommons.org/licenses/by/4.0/), which permits unrestricted use, distribution, and reproduction in any medium, provided you give appropriate credit to the original author(s) and the source, provide a link to the Creative Commons license, and indicate if changes were made. The Creative Commons Public Domain Dedication waiver (http://creativecommons.org/publicdomain/zero/1.0/) applies to the data made available in this article, unless otherwise stated. 


\section{Background}

Endobronchial ultrasound-guided transbronchial needle aspiration (EBUS-TBNA) is a technique that is highly effective for diagnosing enlarged mediastinal and hilar lymph nodes (LNs) detected on computed tomography (CT) or positron emission tomography-CT (PET-CT), in patients with benign or malignant conditions. Some clinical studies have demonstrated that EBUS-TBNA is a cost-effective [1] and safe diagnostic technique for acquiring specimens, with a diagnostic yield similar to or even higher than that of surgical mediastinoscopy [2-4]. However, the optimal procedure of EBUS-TBNA for maximizing the diagnostic yield and minimizing the procedural complexity is controversial. In the past decade, various modifications of the EBUS-TBNA procedure [5-9] have been described for optimizing diagnostic yield, procedural efficiency, and specimen adequacy. Nevertheless, there has been limited discussion on how to simplify the procedure without decreasing its diagnostic yield.

The conventional EBUS-TBNA technique requires that a metal stylet within the inner lumen of a fine needle be inserted and removed during every needle pass, which increases the procedural time and complicates the procedure. Therefore, we considered the possibility of omitting the inner-stylet during EBUS-TBNA. In addition, because there is considerable controversy regarding whether it is necessary to apply suction during EBUSTBNA, we here conducted a prospective randomized controlled trial to determine the optimal EBUS-TBNA procedure for detection of mediastinal and hilar malignant lymphadenopathy, with a particular focus on the effect of using an inner-stylet and suction.

\section{Methods}

\section{Trial subjects}

Consecutive patients with enlarged mediastinal and hilar LNs who underwent EBUS-TBNA between October 2016 and May 2017 were enrolled. An LN with a shortaxis diameter $>5 \mathrm{~mm}$ on a chest CT image was considered as an enlarged LN. Lymph-node stations were classified in accordance with the international lymphnode map by the International Association for the Study of Lung Cancer [10]. All patients provided written informed consent. The EBUS-TBNA procedure was performed by the same experienced endoscopic physician for all patients. It was performed if an enlarged LN was identified by using a convexprobe echoendoscope (EB-530, FUJIFILM, Tokyo, Japan). The trial protocol was approved by the Clinical Research Ethics Committee of our hospital (YJLCYJ2016-216), and the trial was registered at www.chictr.org.cn (ChiCTR-IOR-17010616).

\section{Trial procedure}

TBNA was performed with a 22-gauge needle (NA201SX-4022, Olympus, Tokyo, Japan) under EBUS and real-time color Doppler guidance with a convex-probe echoendoscope. After puncturing an LN, the fine needle was moved to and fro within the LN 10-20 times and then withdrawn. Each LN was sampled with three needle passes using suction-stylet, suction-no stylet, and styletno suction procedures (each process was performed once). To adjust for the effects of some potential confounding factors, including passes made using different procedures, the order of the procedure for each target site was randomized by a senior biostatistician from the School of Public Health, Wenzhou Medical University, using SAS 9.4 for Windows (Cary, North Carolina State, America).

During the conventional EBUS-TBNA procedure (the suction-stylet group), the inner lumen of the fine needle was first occluded with a metal stylet, which was removed after the needle entered the target LN. Once the stylet was withdrawn, a $20-\mathrm{mL}$ syringe was applied to the needle for providing suction. For the no-stylet procedure, the stylet was omitted throughout the procedure, and the syringe was applied to the needle before the latter was inserted into the working channel of the echoendoscope. In the no-suction procedure, after the needle was inserted into the target LN, the stylet was withdrawn by $10 \mathrm{~cm}$ without using suction. During each pass, the physician assessed the amount of bleeding from the puncture site on the bronchial wall.

Each needle-pass specimen was extruded onto a separate glass slide using a 10-mL air-filled syringe, and a direct smear was made by an experienced EBUS nurse. Rapid on-site cytological evaluation was not performed. The residual contents of the needle from a single LN were flushed into the same container and consolidated by formalin to obtain a single cell block or tissue core for histological examination (in accordance with the handing and preparation procedures for histological specimens of our pathology department). After flushing the needle, the outside of the needle and the stylet were vigorously wiped with sterile gauze to reduce cross contamination between passes. A related on-site trial assistant recorded whether or not a visible tissue core was acquired with each needle pass and calculated the procedural time for each pass from the time of insertion of the fine needle through the working channel of the echoendoscope to the retrieval of the needle during each pass, excluding the time for reinsertion of the stylet into the fine needle. If insufficient specimen for a cell block or tissue core was obtained after the third pass, additional passes were permitted, with the choice of procedures and number of passes left to the discretion of the endoscopic physician.

The smears on glass slide were alcohol-fixed (95\% ethanol) and stained with hematoxylin and eosin. A 
cytopathologist, who was blinded to the procedural order for EBUS-TBNA, characterized each individual needle pass for cytological specimen adequacy and made a specific diagnosis as follows: malignancy, benign (including normal lymphoid tissue and granulomatous inflammation), and inadequate. Specimens with $>40$ lymphocytes per high power field [11] in the more cellular areas of the smeared slide was interpreted as adequate, as were those that exhibited malignant cells. Samples with insufficient diagnostic cellular materials or lymphocytes were deemed inadequate.

\section{Assessment of procedural outcomes}

The final diagnosis of each LN was determined using all available cytological and histological findings from three EBUS-TBNA procedures. The primary outcomes of the trial were the cytological specimen adequacy rate and the diagnostic yield for malignant lymphadenopathy. A sample size of 225 LNs would provide sufficient power for a $10 \%$ non-inferiority margin upon performing noninferiority analysis. The secondary outcomes were tissuecore acquisition rate, procedural time and amount of bleeding during each procedure. The amount of bleeding was categorized on the basis of the following scores: 0 (major hemorrhage, resulting in termination of subsequent procedure); 1 (light hemorrhage that could be stopped using cold saline or norepinephrine); 2 (no or little hemorrhage occurred, even without treatment).

\section{Statistical analysis}

Statistical analysis was performed with the IBM SPSS Statistics Version 22.0 software (Chicago, IL, USA). The analysis evaluated only the first three passes for each LN. Descriptive statistics were used to summarize the characteristics of all patients and all LNs in the trial. Data on continuous variables were presented as mean \pm standard deviation. McNemar tests were performed to determine the difference in specimen adequacy rate, diagnostic yield and tissue-core acquisition rate between suction-stylet and suction-no stylet or suction-stylet and stylet-no suction procedures. The paired t-test was employed to compare procedural time. In addition, the amount of bleeding was analyzed using Wilcoxon's test. A subgroup analysis was performed to determine the association of different EBUS-TBNA procedures with specimen adequacy rate and diagnostic yield, for LNs $>10 \mathrm{~mm}$ or $\leq 10 \mathrm{~mm}$ in diameter. A two-sided $P$ value of $<0.05$ was considered to indicate statistical significance.

\section{Results}

The trial evaluated 97 patients with a total of 255 mediastinal and hilar LNs. Randomization ensured that each third of the suction-stylet, suction-no stylet, and styletno suction passes were first, second, and third passed (Fig. 1). The baseline characteristics of the patients and
LNs are summarized in Table 1 . The mean age of patients was 61.2 years (range, 20-79 years); 71 (73.2\%) of the patients were male. Of 57 inpatients for whom we could record clinical symptoms after the EBUS-TBNA procedure, 9 (15.8\%) had transient fever; these 9 patients recovered within $24 \mathrm{~h}$ without treatment or with temporary antifebrile medication. There were no instances of procedure-related major hemorrhage in the present trial.

Two or three LNs were sampled for most patients. For the $255 \mathrm{LNs}$, the mean short-axis diameter on chest CT images was $14.7 \mathrm{~mm}$, and the most common enlarged $\mathrm{LN}$ stations were $4 \mathrm{R}$ and 7 . The final pathologic diagnoses for the 255 LNs were as follows: 104 malignancies, 144 benign diagnoses, and 7 inadequate samples.

The cytological specimen adequacy rates were 87.1, 88.2 , and $85.9 \%$ in the suction-stylet, suction-no stylet, and stylet-no suction groups, respectively; the corresponding values for diagnostic yield of malignancy were $32.2,31.8$, and $31.0 \%$, respectively, which showed no statistically significant difference among the three groups. The results of statistical analysis of specimen adequacy rate and diagnostic yield are detailed in Table 2. Subgroup analysis did not show a statistically significant association between the EBUS-TBNA procedures and specimen adequacy rate or diagnostic yield for LNs $>10 \mathrm{~mm}$ or LNs $\leq 10 \mathrm{~mm}$ (Table 3 ).

Comparison of secondary outcomes among the three procedures (Table 4) revealed that the use of suction was associated with an increase in the tissue-core acquisition rate (suction-stylet vs. stylet-no suction group; $47.1 \%$ [120/255] vs. $32.5 \%$ [83/255]; $P<0.001$ ). Non-use of the stylet did not decrease or increase the tissue-core acquisition rate (suction-stylet vs. suction-no stylet group: $47.1 \%$ vs. $49.4 \% ; P=0.576$ ). In terms of procedural time, the no-stylet procedure was on average $14 \mathrm{~s}$ shorter than the suction-stylet procedure ( $87.1 \mathrm{~s}$ vs. $101.1 \mathrm{~s} ; P<0.001$ ). However, in terms of amount of bleeding, the use or non-use of suction yielded similar scores; similar results were observed for procedures with and without the stylet.

\section{Discussion}

This prospective randomized controlled trial demonstrated that the traditional procedure of applying suction during EBUS-TBNA did not make a statistically significant difference in cytological specimen adequacy or diagnostic yield of malignant lymphadenopathy, although it increased the rate of tissue-core acquisition for histological examination. Compared to procedures performed with a stylet, not using a stylet did not decrease the specimen adequacy or diagnostic yield. Upon comparing EBUS-TBNA procedures with or without suction and with or without stylet for LNs $\leq 10 \mathrm{~mm}$ and $>10 \mathrm{~mm}$ in short-axis diameter, we found no difference in the adequacy or diagnostic yield of cytological specimens. Although more data may be needed 


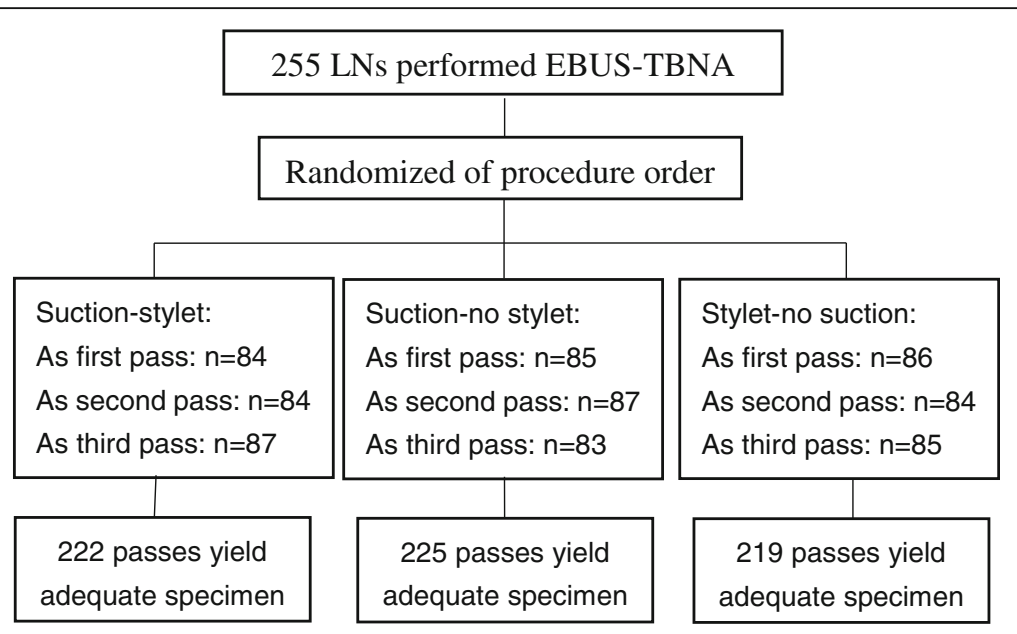

Fig. 1 The number of each pass order and adequate specimen for different EBUS-TBNA procedures. EBUS-TBNA: Endobronchial ultrasound-guided transbronchial needle aspiration; LN: Lymph node

Table 1 Characteristics of patients and lymph nodes included in the final analysis

\begin{tabular}{|c|c|}
\hline Characteristics & Data \\
\hline Patients, No. & 97 \\
\hline Age, years & $61.2 \pm 13.2$ \\
\hline \multicolumn{2}{|l|}{ Gender, No. } \\
\hline Male & 71 \\
\hline Female & 26 \\
\hline \multicolumn{2}{|l|}{ Origin of patient, No. } \\
\hline Outpatient & 40 \\
\hline Inpatient & 57 \\
\hline Lymph nodes, No. & 255 \\
\hline Mean nodule size, mm & $14.7 \pm 7.6$ \\
\hline$\leq 10 \mathrm{~mm}$, No. & 86 \\
\hline$>10$ mm, No. & 169 \\
\hline \multicolumn{2}{|c|}{ Location of lymph nodes, No. } \\
\hline $2 \mathrm{~L}$ & 1 \\
\hline $4 \mathrm{~L}$ & 29 \\
\hline $4 \mathrm{R}$ & 72 \\
\hline 7 & 77 \\
\hline $10 \mathrm{~L}$ & 6 \\
\hline $10 \mathrm{R}$ & 3 \\
\hline $11 \mathrm{~L}$ & 28 \\
\hline $11 \mathrm{R}$ & 26 \\
\hline $12 R$ & 1 \\
\hline mediastinal mass & 12 \\
\hline
\end{tabular}

to confirm these specific conclusions, our findings may assist endoscopic physicians in determining the optimal EBUS-TBNA procedure.

Mediastinal and hilar lymphadenopathy may be caused by various inflammatory, infectious, or malignant factors, and it is important to ascertain the diagnosis or to determine the disease stage in case of malignancy before deciding on treatment. Mediastinoscopy has long been the reference standard of mediastinal and hilar LN sampling; however, it has several disadvantages, including its relatively high complexity and invasiveness [12]. Over the last decade, EBUS-TBNA has provided a more readily available and safer alternative than mediastinoscopy for acquiring specimens $[2,3]$. It has emerged as the best first-diagnostic tool for collecting tissue for diagnosis and staging of lung cancer [13] and has also come to be approved for use in other lymphadenopathies, such as tuberculosis, sarcoidosis and lymphoma [14-16].

However, EBUS-TBNA has several limitations. Although its median sensitivity for detecting malignant lymphadenopathy (89\%) [13] is better than that of imaging examinations alone, EBUS-TBNA leads to misdiagnosis of malignant LNs

Table 2 Comparison of primary outcomes of EBUS-TBNA procedures

\begin{tabular}{lll}
\hline EBUS-TBNA procedure & The primary outcome & $P^{\mathrm{a}}$ value \\
\hline & Specimen adequacy rate & \\
suction-stylet vs. suction-no stylet & $87.1 \%$ vs. $88.2 \%$ & 0.629 \\
suction-stylet vs. stylet-no suction & $87.1 \%$ vs. $85.6 \%$ & 0.728 \\
& Diagnostic yield & \\
suction-stylet vs. suction-no stylet & $32.2 \%$ vs. 31.8\% & $>0.999$ \\
suction-stylet vs. stylet-no suction & $32.2 \%$ vs. 31.0\% & 0.711 \\
\hline $\begin{array}{ll}\text { a: Determined by McNemar test; EBUS-TBNA Endobronchial ultrasound-guided } \\
\text { transbronchial needle aspiration }\end{array}$
\end{tabular}


Table 3 Result of subgroup analysis among EBUS-TBNA procedures for $L \mathrm{Ns}>10 \mathrm{~mm}$ and $\leq 10 \mathrm{~mm}$ in diameter

\begin{tabular}{llll}
\hline Compared & Subgroup & $\mathrm{P}^{\mathrm{a}}$ value & \\
\cline { 3 - 4 } procedures & LN size, $\mathrm{mm}$ & adequacy rate & diagnostic yield \\
\hline suction-stylet vs. & $\leq 10$ & $>0.999$ & $>0.999$ \\
no-stylet & $>10$ & 0.754 & $>0.999$ \\
suction-stylet vs. & $\leq 10$ & $>0.999$ & 0.289 \\
no-suction & $>10$ & 0.523 & $>0.999$ \\
\hline
\end{tabular}

Determined by McNemar test; EBUS-TBNA Endobronchial ultrasound-guided transbronchial needle aspirationm, $L N$ Lymph node

in an average of $11 \%$ of patients. Additionally, physicians need to obtain sufficient tissue specimens from EBUSTBNA for molecular testing for diagnosis of malignancy or microbe cultivation for diagnosis of infectious diseases. Additionally, the EBUS-TBNA technique is time-consuming, especially when multiple LNs are identified and multiple needle passes are made. In our trial, we found that the procedure mainly required an additional $1-3$ min for every needle pass and up to $10-15 \mathrm{~min}$ additionally per $\mathrm{LN}$. Lastly, given the recommendations for combining EBUSTBNA and endoscopic ultrasound-guided fine needle aspiration (EUS-FNA) for diagnosis of mediastinal and hilar lymphadenopathy [17], the operating physician should be skilled in both procedures. Consequently, it is important to simplify the EBUS-TBNA procedure and acquire adequate specimens without decreasing its diagnostic yield.

The use of an inner-stylet during EBUS-TBNA is somewhat controversial. It has commonly been used because it can theoretically prevent bronchial mucosa and cartilage filling the inner lumen and protect the fine needle by increasing its stiffness upon entry into the target LN. However, the inner-stylet has to be inserted and removed through the fine needle during every needle pass, which increases the procedural time and complicates the EBUS-TBNA procedure. Moreover, conventional TBNA is performed without an inner-stylet in the fine needle. Evaluation of the use of the inner-stylet in EBUS-TBNA has been limited to a single recent study. Scholten and colleagues [18] found no significant differences in diagnostic yield, specimen adequacy, or cytological quality between with-stylet and no-stylet procedures; these conclusions agreed with our findings. However, these previous authors failed to quantify the procedural time saved by omitting the stylet. In our trial, we found that non-use of the stylet statistically significantly decreased the procedural time; relative to the suction-stylet procedure, the no-stylet procedure was on an average $14 \mathrm{~s}$ shorter, excluding the time spent on inserting the inner-stylet into the fine needle. In addition, during the entire trial, there was no instance of needle breakage when the inner-stylet was not used. Moreover, from the patient's perspective, omitting the inner-stylet might help reduce the cost of EBUS needles. Although the clinical value of the time saved with the no-stylet procedure merits further study, it is evident that omitting the stylet could simplify the EBUS-TBNA procedure, without reducing the cytological specimen adequacy or diagnostic yield.

Application of suction during FNA has been a standard practice for many decades in various medical specialties, including pathology and gastroenterology. However, there is considerable controversy about the need to apply suction during EBUS-TBNA. Some clinicians believe that suction might increase tissue trauma at the biopsy site and result in more bleeding into the specimen, thus decreasing the diagnostic yield of EBUS-TBNA. Others have argued that suction helps to acquire more specimen material. Wallace et al. [19] reported that, compared to FNA without suction, the traditional method of applying suction during EUS-FNA did not show any difference in diagnostic yield but provided worse specimen quality because of excessive blood in the specimen. Recently, Casal et al. [20] conducted a randomized trial for comparing the with-suction and no-suction procedures of EBUSTBNA and found no difference in diagnostic yield, adequacy or quality of cytological specimens. However, they did not analyze the histological specimen adequacy of each procedure. A retrospective nonrandomized study showed that high suction pressures during EBUS-TBNA might be useful for safe collection of sufficient tissue specimens [21]. Our trial data support the conclusion that suction does not influence cytological specimen adequacy, diagnostic yield or the amount of bleeding.

Rapid advances in oncologic therapy have necessitated further ancillary studies, including immunohistochemical

Table 4 Statistical results of the three procedures in secondary outcomes

\begin{tabular}{|c|c|c|c|c|c|}
\hline \multirow[t]{2}{*}{ Secondary outcome } & \multirow[t]{2}{*}{ Group $A^{b}$} & \multirow[t]{2}{*}{ Group Bc } & \multirow[t]{2}{*}{ Group $C^{d}$} & \multicolumn{2}{|l|}{$P$ value ${ }^{a}$} \\
\hline & & & & A vs. B & A vs. C \\
\hline Procedural time (second) & $101.1 \pm 31.3$ & $87.1 \pm 34.7$ & $89.3 \pm 33.6$ & $<0.001$ & $<0.001$ \\
\hline Tissue-core acquisition (\%) & 47.1 & 49.4 & 32.5 & 0.576 & $<0.001$ \\
\hline The amount of bleeding (score) & $1.97 \pm 0.17$ & $1.96 \pm 0.20$ & $1.97 \pm 0.17$ & 0.366 & $>0.999$ \\
\hline
\end{tabular}

aprocedural time, tissue-core acquisition rate, and the amount of bleeding were analyzed by the paired t-test, McNemar test, and the Wilcoxon's test, respectively

${ }^{\mathrm{b}} \mathrm{A}$, suction-stylet procedure

${ }^{\mathrm{C}} \mathrm{B}$, suction-no stylet procedure

${ }^{\mathrm{d} C}$, stylet-no suction procedure 
and molecular analyses for subtyping and genotyping of lung cancer, during the diagnostic workup of small tissue specimens. A guideline from the College of American Pathologists, International Association for the Study of Lung Cancer, and Association for Molecular Pathology, states that tissue samples should be prioritized for molecular analysis and that cytological samples are also suitable for studies, with cell blocks being preferred over smeared material [22]. In our trial, the suction procedure assisted in obtaining a greater volume of tissue specimens, which could be processed for cell block or tissue histology analyses. However, another guideline for the acquisition and preparation of EBUS-TBNA specimens for the diagnosis of lung cancer suggests that cell blocks and core tissue are both good materials for mutational analysis [23]. Numerous studies have reported on preparation of cell blocks for ancillary studies [24-26]. Therefore, further investigations may be needed to explore whether the amount of tissue-core obtained during EBUS-TBNA with or without suction would influence the diagnosis and subtyping of lung cancer.

The most frequent complication associated with EBUSTBNA is hemorrhage; other rare complications of the procedure are infection, pneumothorax, and device breakage [27]. In 2014, a systematic review on adverse events in 16,181 patients who underwent endosonography for mediastinal and hilar LNs or central lung masses reported $23(0.14 \%)$ serious adverse events $(0.3$ and $0.05 \%$ with EUS-FNA and EBUS-TBNA, respectively), with no mortality [28]. In the present trial, there were no instances of severe infectious disease, need for ICU admission, or death after EBUS-TBNA. The incidence of fever was $15.8 \%$ (9/57) among inpatients in our trial, and all febrile patients recovered in $24 \mathrm{~h}$ without treatment or with temporary antifebrile medication. In addition, there was no significant difference in the amount of bleeding with each pass between EBUS-TBNA procedures with and without suction or with and without stylet, and there was no instance of major hemorrhage. These results suggest that EBUS-TBNA is a safe method in general and that the probability of complications is similar among the different EBUS-TBNA procedures.

An advantage of the present prospective trial, which involved randomization of the procedure order and blinding of the cytopathologist, is its self-contrast design, which could control for the effects of size, location, density, and pathological type of different LNs, as well as other unknown factors. Furthermore, in our trial, an on-site assistant recorded the procedural time for each pass; this has not been evaluated in other previous studies.

A limitation of our trial, however, is its single-centre and single-operator design. A multicentre trial would be ideal to confirm the statistical significance of the results obtained with different EBUS-TBNA procedures.
Additionally, we compared tissue-core acquisition rate among different procedures but, regrettably, failed to analyze the specimen quality for cell block or tissue histological examination, which might have decreased the chance of identifying differences among the procedures.

\section{Conclusions}

In summary, the use of suction or non-use of the inner-stylet does not make a significant difference in cytological specimen adequacy or diagnostic yield when performing EBUS-TBNA. Omitting the stylet can simplify the procedure, and applying suction can help increase the tissue-core acquisition rate. These findings may assist endoscopic physicians in determining the optimal EBUS-TBNA procedure and warrant clinical verification in a future multicentre study.

\section{Abbreviations}

CT: computed tomography; EBUS-TBNA: endobronchial ultrasound-guided transbronchial needle aspiration; EUS-FNA: endoscopic ultrasound-guided fine needle aspiration; LN: lymph node; PET-CT: positron emission tomography-computed tomography

\section{Acknowledgements}

We would like to thank Prof. Guangyun Mao from the School of Public Health, Wenzhou Medical University, for his assistance with statistical analysis, and acknowledge all medical workers from the department of Endoscopy and the department of Pathology, The First Affiliated Hospital of Wenzhou Medical University, for their coordination to carry out the clinical trial.

\section{Funding}

The collection of date was supported by grants from the International Pulmonary Key Laboratory of Zhejiang Province to CSC, the Interventional Pulmonology Key Laboratory of Wenzhou City to CSC, the Interventional Pulmonology Innovation Subject of Zhejiang Province to CSC, the National Nature Science Foundation of China (81270313 to CSC, 81770074 to CSC, 81570075 to (SC), the Natural Science Foundation of Zhejiang Province (LZ15H010001 to CSC), the Science Technology Department Foundation of Zhejiang Province (WKJ-ZJ-1526 to CSC) and the National Key R\&D Program of China (2016YFC1304000 to CSC).

\section{Availability of data and materials}

The datasets used and/ or analyzed during the current study are available from the corresponding author on reasonable request.

\section{Authors' contributions}

$\mathrm{XXL}$ analyzed data and was a major contributor in writing the manuscript; CSC designed the trial and analyzed data; MY performed the trial. YPL and KPW designed the trial. JR, QYL, YYL and XHJ collected data; all authors read and approved the final manuscript.

\section{Ethics approval and consent to participate}

The trial was approved by the Clinical Research Ethics Committee of the First Affiliated Hospital of Wenzhou Medical University (YJLCYJ2016-216), and all patients provided written informed consent.

\section{Consent for publication}

Not applicable.

\section{Competing interests}

The authors declare that they have no competing interests.

\section{Publisher's Note}

Springer Nature remains neutral with regard to jurisdictional claims in published maps and institutional affiliations. 


\section{Author details}

'Department of Pulmonary and Critical Care Medicine, The First Affiliated Hospital of Wenzhou Medical University, Ouhai District, Wenzhou, China. ${ }^{2}$ Department of Pathology, The First Affiliated Hospital of Wenzhou Medical University, Ouhai District, Wenzhou, China. ${ }^{3}$ Department of Endoscopy, The First Affiliated Hospital of Wenzhou Medical University, Ouhai District, Wenzhou, China. ${ }^{4}$ Division of Pulmonary and Critical Care Medicine, Johns Hopkins University School of Medicine, Baltimore, USA.

Received: 20 August 2017 Accepted: 22 November 2018

Published online: 07 December 2018

\section{References}

1. Rintoul RC, Glover MJ, Jackson C, et al. Cost effectiveness of endosonography versus surgical staging in potentially resectable lung cancer: a health economics analysis of the ASTER trial from a European perspective. Thorax. 2014;69:679-81.

2. Annema JT, van Meerbeeck JP, Rintoul RC, et al. Mediastinoscopy vs endosonography for mediastinal nodal staging of lung cancer: a randomized trial. JAMA. 2010;304:2245-52

3. Sehgal IS, Dhooria S, Aggarwal AN, et al. Endosonography versus Mediastinoscopy in mediastinal staging of lung Cancer: systematic review and meta-analysis. Ann Thorac Surg. 2016;102:1747-55.

4. Ernst A, Anantham D, Eberhardt R, et al. Diagnosis of mediastinal adenopathy-real-time endobronchial ultrasound guided needle aspiration versus mediastinoscopy. J Thorac Oncol. 2008:3:577-82.

5. Muthu V, Gupta N, Dhooria S, et al. A prospective, randomized, double-blind trial comparing the diagnostic yield of 21- and 22-gauge aspiration needles for performing endobronchial ultrasound-guided Transbronchial needle aspiration in sarcoidosis. Chest. 2016;149:1111-3.

6. Konge L, Clementsen PF, Ringsted C, et al. Simulator training for endobronchial ultrasound: a randomised controlled trial. Eur Respir J. 2015; 46:1140-9.

7. Lee HS, Lee GK, Lee HS, et al. Real-time endobronchial ultrasound-guided transbronchial needle aspiration in mediastinal staging of non-small cell lung cancer: how many aspirations per target lymph node station? Chest. 2008:134:368-74.

8. Casal RF, Lazarus DR, Kuhl K, et al. Randomized trial of endobronchial ultrasound-guided transbronchial needle aspiration under general anesthesia versus moderate sedation. Am J Respir Crit Care Med. 2015;191: 796-803.

9. Guo H, Liu S, Guo J, et al. Rapid on-site evaluation during endobronchial ultrasound-guided transbronchial needle aspiration for the diagnosis of hilar and mediastinal lymphadenopathy in patients with lung cancer. Cancer Lett. 2016;371:182-6.

10. Rusch WW, Asamura $H$, Watanabe $H$, et al. The IASLC lung cancer staging project: a proposal for a new international lymph node map in the forthcoming seventh edition of the TNM classification for lung cancer. Thorac Oncol. 2009;4:568-77.

11. Alsharif M, Andrade RS, Groth SS, et al. Endobronchial ultrasound-guided transbronchial fine-needle aspiration: the University of Minnesota experience, with emphasis on usefulness, adequacy assessment, and diagnostic difficulties. Am J Clin Pathol. 2008;130:434-43.

12. Detterbeck FC, Jantz MA, Wallace $M$, et al. Invasive mediastinal staging of lung cancer: ACCP evidence-based clinical practice guidelines (2nd edition). Chest. 2007:132:202S-20S.

13. Silvestri GA, Gonzalez AV, Jantz MA, et al. Methods for staging non-small cell lung cancer: diagnosis and management of lung cancer, 3rd ed: American College of Chest Physicians evidence-based clinical practice guidelines. Chest. 2013;143:e211S-50S.

14. Navani N, Molyneaux PL, Breen RA, et al. Utility of endobronchial ultrasound-guided transbronchial needle aspiration in patients with tuberculous intrathoracic lymphadenopathy: a multicentre study. Thorax. 2011;66:889-93.

15. Davis GS. The role of endobronchial ultrasound-guided transbronchial needle aspiration (EBUS TBNA) in the diagnosis of sarcoidosis. Cancer Cytopathol. 2014;122:239-40

16. Kheir F, Itani A, Assasa O, et al. The utility of endobronchial ultrasound-transbronchial needle aspiration in lymphoma. Endosc Ultrasound. 2016:5:43-8.
17. Vilmann $P$, Clementsen PF, Colella $S$, et al. Combined endobronchial and esophageal endosonography for the diagnosis and staging of lung cancer: European Society of Gastrointestinal Endoscopy (ESGE) guideline, in cooperation with the European Respiratory Society (ERS) and the European Society of Thoracic Surgeons (ESTS). Endoscopy. 2015;47:545-59.

18. Scholten EL, Semaan R, Illei P, et al. Stylet use does not improve diagnostic outcomes in endobronchial Ultrasonographic Transbronchial needle aspiration: a randomized clinical trial. Chest. 2017;151:636-42.

19. Wallace MB, Kennedy T, Durkalski V, et al. Randomized controlled trial of EUS-guided fine needle aspiration techniques for the detection of malignant lymphadenopathy. Gastrointest Endosc. 2001;54:441-7.

20. Casal RF, Staerkel GA, Ost D, et al. Randomized clinical trial of endobronchial ultrasound needle biopsy with and without aspiration. Chest. 2012;142:568-73.

21. Shiroyama T, Okamoto N, Suzuki H, et al. Usefulness of high suction pressure for sufficient tissue collection during endobronchial ultrasound guided transbronchial needle aspiration. PLoS One. 2013;8:e82787.

22. Lindeman $\mathrm{NI}$, Cagle PT, Beasley MB, et al. Molecular testing guideline for selection of lung cancer patients for EGFR and ALK tyrosine kinase inhibitors: guideline from the College of American Pathologists, International Association for the Study of Lung Cancer, and Association for Molecular Pathology. J Thorac Oncol. 2013;8:823-59.

23. van der Heijden EH, Casal RF. Trisolini Rocco et al. Guideline for the acquisition and preparation of conventional and endobronchial ultrasoundguided transbronchial needle aspiration specimens for the diagnosis and molecular testing of patients with known or suspected lung cancer. Respiration. 2014:88(6):500-17.

24. Neal N, Brown James M, Matthew N, et al. Suitability of endobronchial ultrasound-guided transbronchial needle aspiration specimens for subtyping and genotyping of non-small cell lung cancer: a multicenter study of 774 patients. Am J Respir Crit Care Med. 2012;185(12):1316-22.

25. Abhishek B, Leon Marino E, Peter D, et al. Clinical performance of endobronchial ultrasound-guided transbronchial needle aspiration for assessing programmed death ligand-1 expression in nonsmall cell lung cancer. Diagn Cytopathol. 2018;46(5):378-83.

26. Emily $\mathrm{H}$, David $\mathrm{M}$, lan $\mathrm{P}$, et al. Cell block samples from endobronchial ultrasound transbronchial needle aspiration provide sufficient material for ancillary testing in lung cancer-a quaternary referral centre experience. J Thorac Dis. 2016;8(9):2544-50.

27. Asano F, Aoe M, Ohsaki Y, et al. Complications associated with endobronchial ultrasound-guided transbronchial needle aspiration: a nationwide survey by the Japan Society for Respiratory Endoscopy. Respir Res. 2013:14:50

28. von Bartheld MB, van Breda A, Annema JT. Complication rate of endosonography (endobronchial and endoscopic ultrasound): a systematic review. Respiration. 2014;87:343-51.

\section{Ready to submit your research? Choose BMC and benefit from:}

- fast, convenient online submission

- thorough peer review by experienced researchers in your field

- rapid publication on acceptance

- support for research data, including large and complex data types

- gold Open Access which fosters wider collaboration and increased citations

- maximum visibility for your research: over $100 \mathrm{M}$ website views per year

At $\mathrm{BMC}$, research is always in progress.

Learn more biomedcentral.com/submission 